\title{
Pictorial interference with geometric shapes and achromatic shades
}

\author{
RICHARD P. COMPTON and JOHN H. FLOWERS \\ University of Nebraska-Lincoln, Lincoln, Nebraska 68588
}

\begin{abstract}
In two experiments, subjects were required to match printed names with subsequently displayed achromatic shades or geometric shapes. The presence of incongruent shades or shapes at the time of word display substantially slowed the RT for performing the match. This pictorial interference (interference due to a visual, nonlinguistic attribute) was substantially attenuated, however, as the delay between the word display and shapes or shades to be matched was increased beyond $100 \mathrm{msec}$. These findings illustrate the occurrence of a transitory automatic pathway activation from an irrelevant pictorial attribute, which may be contrasted with the verbal interference effects typically observed in Stroop-naming tasks. In both our tasks and the Stroop task, selective attention fails when a stimulus must be transformed to another form of encoding in the presence of a competing stimulus which is already close to that form.
\end{abstract}

In the traditional Stroop task, the naming of the color of the ink in which a word is printed is facilitated or interfered with by the content of the word itself (Stroop, 1935). Dyer (1973) and Jensen and Rohwer (1966) have reviewed a variety of tasks that produce similar forms of verbal interference (interference deriving from an irrelevant linguisticverbal attribute). In these cases, an unwanted reading response produces interference in tasks requiring a naming response. Fewer studies have been directed at situations where interference arises from a pictorial (nonlinguistic) attribute.' In fact, the presence of an incongruent pictorial attribute appears to have little, if any, interfering effect in studies involving an oral response on the part of the subject. For example, Stroop (1935) found only negligible interference from an irrelevant color on the reading of color words.

This asymmetry in interference (in which the effect produced by irrelevant colors on a word-reading response appears to be substantially less than that resulting from words in a task requiring a naming response) seems to occur only in tasks requiring an overt oral response. Interference from an irrelevant pictorial attribute has been found in such varied tasks as judging the direction in which arrows point (Clark \& Brownell, 1975), word scanning (Uleman \& Reeves, 1971), same-different matching (Dyer, 1973; Treisman \& Fearnley, 1969), and word-color matching (Flowers, 1975). Apparently the occurrence

A portion of this research was presented at the 48th Annual Meeting of the Midwestern Psychological Association, May 7, 1976. This research was supported by a grant from the University of Nebraska Research Council, including funding from NIH Grant RR-07055-09 to the University of Nebraska. The authors thank Rosemary Lyons for assistance in running Experiment 2. of pictorial interference is a function not only of the presence of incongruent pictorial attributes, but also of the form of encoding necessary for making the required response.

\section{Form of Coding: Stimulus and Response}

Treisman and Fearnley (1969), in attempting to account for a variety of pictorial and verbal interference phenomena, have suggested that interference results when a perceptual task requires the transformation of a stimulus into the form of representation most suitable for making the required response in the presence of a competing stimulus that is already closer to the desired form of encoding. According to this model, interference in the traditional Stroop task results from the need to transform a pictorial attribute (ink color) into a verbal form of encoding (name) in the presence of a written color name. Flowers (1975) reported finding a reverseStroop effect (i.e., interference from the pictorial attribute-ink color) in a task requiring the matching of a word (a color-name) to its pictorial form of encoding in the presence of an incongruent pictorial attribute. Both of these tasks (Stroop and reverseStroop) require a transformation of the form of encoding of the relevant stimulus information. In other tasks where the form of encoding from stimulus to response remains the same (i.e., a transformation from a verbal to pictorial code is not necessary), little or no interference occurs (Egeth, Blecker, \& Kamlet, 1969).

Table 1 shows the four different conditions that can occur in tasks involving both pictorial and verbal sources of information, depending on whether the relevant stimulus information is contained in the verbal or pictorial attribute, and whether the response requires oral naming (reading) or some varia- 


\begin{tabular}{lcc} 
& $\begin{array}{c}\text { Table 1 } \\
\text { Occurrence of Interference for the Different } \\
\text { Conditions in Pictorial-Verbal Tasks }\end{array}$ \\
\hline $\begin{array}{l}\text { Relevant } \\
\text { Stimulus }\end{array}$ & \multicolumn{2}{c}{ Response Mode } \\
\cline { 2 - 3 } Attribute & Naming & Matching \\
\hline Pictorial & $\begin{array}{c}\text { 1. Interference } \\
\text { (from words) }\end{array}$ & $\begin{array}{c}\text { 2. No Interference } \\
\text { (from words) }\end{array}$ \\
Verbal & $\begin{array}{c}\text { 3. No Interference } \\
\text { (from pictorial attributes) }\end{array}$ & $\begin{array}{c}\text { 4. Interference } \\
\text { (from pictorial attributes) }\end{array}$ \\
\hline
\end{tabular}

tion on pictorial matching. The table indicates whether interference occurs in the different conditions. These conditions are (clockwise from the top left):

Pictorial attribute relevant-oral naming response. The classic Stroop task, where interference results from the presence of an incongruent verbal attribute in naming the relevant visual attribute, is a prime example of this condition (Stroop, 1935). This effect has been found for a variety of stimulus dimensions besides color, including numerosity (Morton, 1969), direction (Shor, 1970; White, 1969), typeface (Warren \& Lasher, 1974), and achromatic shades (Dyer, 1971).

Pictorial attribute relevant-pictorial matching response. In this condition no interference results from the presence of an incongruent verbal attribute. Egeth, Blecker, and Kamlet (1969) found no interference in a matching task when color patches were embossed with incongruent color names, and nor did Treisman and Fearnley (1969) in a similar matching task. Uleman and Reeves (1971) also found no interference from words on a visual scanning task.

Verbal attribute relevant-verbal naming (reading) response. Virtually no interference is found from an incongruent visual attribute when the task requires naming (reading) the words. Dyer and Severance (1972) found an increase in response time of less than $3 \%$ for reading color-words printed in incongruent ink colors compared to when they were printed in gray ink. ${ }^{2}$

Verbal attribute relevant-pictorial matching response. Both Dyer (1973) and Treisman and Fearnley (1969) have reported finding interference in same-different matching tasks from an incongruent pictorial attribute, but the simultaneous presentation of words and colors in both experiments makes it difficult to determine the form of encoding actually used in the comparison, since either stimulus could have been transformed prior to responding. Uleman and Reeves (1971) have also reported interference in a scanning task from incongruent colors, but since they had their subjects scan for a single specific word, mark it, and name it (incorporating both a scanning and naming response in the same task), it is again difficult to interpret their results in the present framework. Perhaps one of the clearest examples of this condition is given in a study by Flowers (1975) in which he reported finding interference when an incongruent pictorial attribute was present in a word-color matching task. Flowers had his subjects match a previously displayed word to a subsequently displayed color.

\section{Dimensions Other Than Color}

Most previous studies on pictorial interference have used the pictorial attribute of color (Dyer \& Severance, 1972; Flowers, 1975; Gumenick \& Glass, 1970; Treisman \& Fearnley, 1969; Uleman \& Reeves, 1971); an exception is the recent study by Clark and Brownell (1975), which dealt with stimuli where both attributes were pictorial. Yet, if the explanation of Treisman and Fearnley for pictorial and verbal interference is truly general, then in a matching task like that used by Flowers (1975), a similar form of pictorial interference should occur for stimulus dimensions other than color. Dyer (1973) has previously suggested that the processing color information is somehow different from the processing of information about other dimensions, since dimensions other than color appear to be less subject to interference in the traditional Stroop task. According to Dyer, color seems to be a special type of stimulus dimension on which attention cannot be focused exclusively to the complete attenuation of the irrelevant words (in the Stroop task), whereas achromatic shades, direction, numerosity, etc., do allow considerable focusing of attention with the resultant partial attenuation of the irrelevant word (Dyer, 1973). This difference may be due to the other dimensions' being more spatially separable from the verbal attribute. For example, Flowers and Stoup (1977) have shown that interference from irrelevant words can be eliminated when the pictorial dimensions are physically separated from the words (shape or color in their study).

It seems possible therefore that in a word-picture matching task, dimensions other than color (especially those that are more spatially separated from the verbal attribute) may not lead to analogous forms of pictorial interference. Thus, one purpose of the present experiments was to determine whether a similar form of pictorial interference would occur in a word-picture matching task for the pictorial dimensions of achromatic shade and geometric shape. The occurrence of pictorial interference would support the theory of Treisman and Fearnley (1969) and would suggest that no differences exist (at least for this task) in a subject's ability to selectively attend to color, shade, or shape.

\section{Delay Interval}

A related question concerns the decrease in the 
amount of pictorial interference found by Flowers (1975) in his study when the interval between the presentation of the color-name (presented for $100 \mathrm{msec}$ ) and the matching field (containing color patches) was varied between 20 and $700 \mathrm{msec}$. He found interference from the pictorial attribute only at the brief intervals (100 msec or less) with attenuation of the interference at the longer intervals. Previous research (Posner, Boies, Eichelman, \& Taylor, 1969; Tversky, 1969) has shown that in tasks requiring the matching of sequentially displayed stimuli presented in different forms, the interval is used to transform the information from the first stimulus into a form suitable for a rapid comparison with the second stimulus. On that basis, Flowers interpreted the interference effect in his study as a result of competition between the irrelevant pictorial attribute and the pictorial encoding of the relevant verbal attribute. If this interpretation is correct, then a comparison of the effect of the delay interval between the word and pictorial matching field in the present experiments with the data described by Flowers for color would provide information about the time course for encoding these dimensions. While achromatic shade is similar to color in being a continuous dimension and could therefore be expected to lead to a similar attenuation of interference with longer delays, geometric shapes form a quite different qualitative dimension and might be expected to be processed differently.

\section{METHOD}

Since the general procedure for both experiments to be reported was essentially the same, a general description will be given that applies to both. The specific differences will then be described. In both experiments, the subjects performed a manual matching task, in which they were required to press a "right" or "left" response key indicating which of the two simultaneously presented pictorial stimuli had been named by a previously presented word. In both experiments the interval between the stimulus word and the choice of pictorial stimuli was varied between 20,100 , and $300 \mathrm{msec}$. Each trial consisted of the sequential presentation of a word field and a matching field.

Following an oral ready signal from the experimenter, each experimental trial began with the presentation of a stimulus word for $100 \mathrm{msec}$, followed by a blank interval $(20,100$, or $300 \mathrm{msec})$, followed by a matching field for $2.0 \mathrm{sec}$. The subjects were instructed to respond "as rapidly as possible without making errors" after the onset of the matching field by pressing the appropriate response key that corresponded to the position of the pictorial stimulus named by the previous stimulus word. RTs were measured as the time in milliseconds from the onset of the matching field to the depression of the response key. The subjects were notified if an error occurred; no other feedback was given.

\section{Experiment 1}

Subjects. Six University of Nebraska students (three male, three female) served as volunteer subjects and were run individually in four 1-h sessions on consecutive days. They were all right-handed and had normal visual acuity.

Stimulus materials. There were four stimulus word cards containing either the word "GRAY" or the word "BLACK" printed in white ink in capital letters approximately $.6 \mathrm{~cm}$ high. Each word was printed both on a black background and on a gray background, such that each word occurred on both a congruent background and an incongruent background. ${ }^{3}$ The background patches were $3.7 \times 5.0 \mathrm{~cm}$. Approximate Munsell value notations of the gray and black backgrounds were $v=6$ and $v=4$. The stimuli were centered on white cards approximately $12.7 \times$ $17.8 \mathrm{~cm}$ in size and occupied about $1.9 \mathrm{deg}$ of visual angle.

The matching field contained a rectangular patch of gray and one of black centered on points $4.0 \mathrm{~cm}$ to the right and to the left of the geometric center of the card (or about $1.8 \mathrm{deg}$ of visual angle from the center of the field). On one-half of the trials, the gray patch appeared on the left and the black patch on the right; these positions were reversed on the other half of the trials.

Apparatus. Stimuli were displayed in a Scientific Prototype Model GB three-channel tachistoscope. The subjects were seated in front of the viewing hood at a table on which were located three telegraph keys. The center key was a start key which initiated the display sequence, the left and right keys were for the response.

Procedure. Each trial was initiated by the subjects pressing the center start key. Prior to the depression of the start key, a fixation field was present containing a small dot in the center. During the delay interval a blank field was presented. The fixation, stimulus, blank, and matching fields were of equal brightness (about $25 \mathrm{fL}$ ). The three delay intervals were run in blocks of 45 trials at each of the four experimental sessions. The first experimental session was treated as a practice session and only the data from the last three sessions were included in the analysis. The order of the delay intervals within a session for the last 3 days was determined by a 3 by 3 Latin square, such that each subject received a different order on a different day. The order of presentation of delays was also counterbalanced across subjects. Within each block of 45 trials, the first five of which were practice, each word-shade combination occurred 10 times (e.g., GRAY on a gray background). Right and left responses to each word-shade combination occurred equally often.

\section{Experiment 2}

Subjects. Thirty-six University of Nebraska students participated as a means of fulfilling a course requirement. They were all righthanded and had normal visual acuity. The subjects were run in groups of three, in a single session lasting about $1 \frac{1}{2} \mathrm{~h}$.

Stimulus materials. The stimuli used were $35-\mathrm{mm}$ slides. The word stimuli contained either the word "CIRCLE" or the word "SQUARE" presented alone or surrounded by a circle or a square (see Figure 1). This resulted in six different stimuli, with each of the two words occurring alone, with the congruent shape, and with the incongruent shape. When projected on a viewing screen, the letters (and shapes surrounding the letters if present) appeared black, the letters approximately $6.5 \mathrm{~cm}$ in height, and the surrounding shapes approximately $39 \mathrm{~cm}$ in diameter for the circle and $42 \mathrm{~cm}$ high for the square (visual angle equivalents were, respectively, $1.0^{\circ}, 4.5^{\circ}$, and $4.8^{\circ}$ ).

Apparatus. Stimuli were projected on a screen by means of two Kodak Carousel projectors combined with Lafayette tachistoscopic shutters. The subjects were seated at three experimental booths located at a viewing distance of approximately $5 \mathrm{~m}$ from the projection screen. Each booth contained two response keys. The room was dimly lit by a $15-\mathrm{W}$ incandescent lamp located in a control booth behind the subjects.

Procedure. There were 12 subjects run at each of the three delay intervals. For one group, the delay between the of fset of the word field and the onset of the matching field was $20 \mathrm{msec}$, for another group the delay was $100 \mathrm{msec}$, and for the last group it was $300 \mathrm{msec}$. Each trial was initiated by the experimenter following the oral ready signal.

Each group of three subjects being run at one time received an initial block of 48 practice trials. After a 5 -min break, they received three blocks of 64 trials with 5-min breaks separating the blocks. The initial four trials of each of the three subsequent 


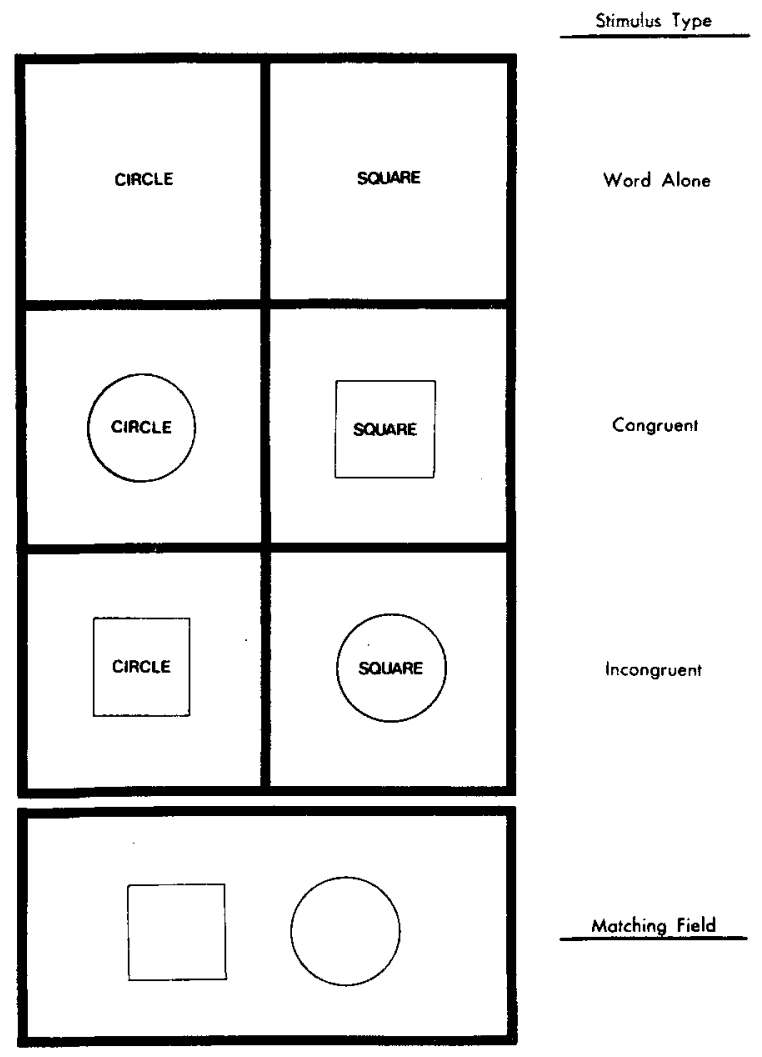

Figure 1. Examples of stimuli used in Experiment 2 showing word-alone, congruent, and incongruent word stimuli. An example of a matching field is shown at the bottom.

blocks were considered practice, leaving a total of 180 experimental trials. For the remaining 60 trials of each block, each of the six word-shape stimulus combinations occurred 10 times. For each word-shape stimulus combination, "left" and "right" responses were required equally often. Within each block of trials, the stimuli were arranged randomly, with the restriction that no stimulus occur consecutively.

\section{RESULTS}

\section{Experiment 1}

Table 2 shows the mean RTs occurring for each word-shade combination at each delay interval. Analysis of variance revealed a significant main effect for conditions $[F(1,5)=17.8, p<.01]$, with the incongruent stimuli producing much longer response times. The Delays by Conditions interaction was also significant $[\mathrm{F}(2,10)=21.3, \mathrm{p}<.01]$, with the difference between the two conditions decreasing as the delay interval increased. The average differences were, for the $20-, 100-$, and $300-\mathrm{msec}$ delays, respectively, 55, 41 , and $13 \mathrm{msec}$. Clearly, the trend is for the amount of interference to decrease with longer delays. Specific comparisons among the two word-shade combinations at the three levels of delay produced significant differences for the 20 - and 100 -msec delays $[F(1,5)=20.7, \mathrm{p}<.01$, and $F(1,5)$
$=18.1, \mathrm{p}<.01]$ while indicating a marginal effect at the 300 -msec delay $[F(1,5)=6.18, p=.05]$.

A further breakdown of the data shows that the same ordering of latencies occurred whether the stimulus word was "GRAY" or "BLACK." No significant interaction resulted between word-shade combination and which word was presented $(\mathrm{F}<1.0)$.

The standard errors and proportion of errors occurring in each condition at each delay interval are also listed in Table 2 . While the error rates were too low to allow for a meaningful statistical analysis, it should be noted that a higher error rate occurred for the incongruent condition and that the rate declined as the delay increased. This pattern completely complements the RT data. These data clearly indicate a considerable influence of the visual attribute (background shade) on the matching latency when the delay between the word and matching field is sufficiently brief.

\section{Experiment 2}

Table 3 displays the mean RTs, standard errors, and proportion errors occurring in each word-shape combination at each delay interval. Comparisons among the three conditions showed significant differences at all three delay intervals $[20$-msec, $F(2,22)$ $=28.4, \mathrm{p}<.01 ; 100-\mathrm{msec}, \mathrm{F}(2,22)=20.5, \mathrm{p}<.01$; and $300-\mathrm{msec}, F(2,22)=8.3, \mathrm{p}<.01]$, though the magnitude of the interference was considerably reduced at the 300 -msec delay.

Looking at the word alone and incongruent combinations, there were significant differences at all three delays $[\mathrm{F}(1,22)=19.7, \mathrm{p}<.01 ; \mathrm{F}(1,22)=$ $13.4, \mathrm{p}<.01 ; \mathrm{F}(1,22)=5.6, \mathrm{p}<.05$, for the 20 -,

Table 2

Mean RT (msec), Standard Error (SE), and Proportion Error (PE) for Each Word Shade Combination as a Function of Delay

\begin{tabular}{|c|c|c|c|c|c|c|c|c|c|}
\hline \multirow[b]{3}{*}{ Condition } & \multicolumn{9}{|c|}{ Delay } \\
\hline & \multicolumn{3}{|c|}{20} & \multicolumn{3}{|c|}{100} & \multicolumn{3}{|c|}{300} \\
\hline & RT & SE & $\mathrm{PE}$ & $\mathrm{RT}$ & SE & PE & RT & $\mathrm{SE}$ & PE \\
\hline $\begin{array}{l}\text { Congruent } \\
\text { Incongruent }\end{array}$ & $\begin{array}{l}383 \\
438\end{array}$ & $\begin{array}{l}3.8 \\
5.8\end{array}$ & $\begin{array}{l}.01 \\
.10\end{array}$ & $\begin{array}{l}353 \\
394\end{array}$ & $\begin{array}{l}3.8 \\
5.9\end{array}$ & $\begin{array}{l}.02 \\
.08\end{array}$ & $\begin{array}{l}335 \\
348\end{array}$ & $\begin{array}{l}2.8 \\
4.0\end{array}$ & $\begin{array}{l}.02 \\
.04\end{array}$ \\
\hline
\end{tabular}

Table 3

Mean RT (msec), Standard Error (SE), and Proportion Error (PE) for Each Word-Shape Combination as a Function of Delay

\begin{tabular}{|c|c|c|c|c|c|c|c|c|c|}
\hline \multirow[b]{3}{*}{ Condition } & \multicolumn{9}{|c|}{ Delay } \\
\hline & \multicolumn{3}{|c|}{20} & \multicolumn{3}{|c|}{100} & \multicolumn{3}{|c|}{300} \\
\hline & RT & SE & $\mathrm{PE}$ & RT & SE & PE & RT & SE & PE \\
\hline Word Alone & 452 & 7.6 & .08 & 385 & 5.6 & .06 & 364 & 4.5 & .02 \\
\hline Congruent & 468 & 7.9 & .07 & 386 & 4.8 & .03 & 374 & 5.1 & .01 \\
\hline Incongruent & 544 & 11.4 & .08 & 429 & 5.7 & .07 & 393 & 5.8 & .07 \\
\hline
\end{tabular}


100-, and 300-msec delays, respectively]. There were no differences between the congruent combinations and either the incongruent or word-alone stimuli at any of the three delays $(F<1.0)$. As in Experiment 1, no differences developed for the two different stimulus words "CIRCLE" or "SQUARE." Again, the pattern of errors compliments the RT data, with the exception that fewer errors occurred for the congruent condition than for the word-alone condition.

The RT data show that the presence of an incongruent shape surrounding a shape-name considerably slows the matching latency between a word and the corresponding shape. The absence of any difference between the word alone and congruent conditions suggest that no facilitation resulted from the congruence between the visual and verbal attributes. ${ }^{4}$ As in the previous experiment, the amount of interference produced by the incongruent shape decreased with the longer delay intervals.

\section{DISCUSSION}

Both experiments indicate that interference results when an incongruent pictorial attribute is present in a task requiring a match between a verbal stimulus attribute and its pictorial form of representation. In both cases, it appears that a failure to gate the incongruent pictorial attribute produces interference at some point in the sequence of processing the verbal attribute prior to the actual response.

Posner and Snyder (1975a) have suggested that Stroop-like interference effects occurring in tasks employing stimuli with more than one attribute dimension (i.e., pictorial and verbal) may be understood by recognizing that the subjects cannot avoid processing the different attributes of the stimulus. In the traditional Stroop task, the word, which is the irrelevant dimension, automatically initiates unwanted processing. Since words are read faster than colors are named (Fraisse, 1969), output interference results when a verbal response is required. Similarly, in the present task, the shade or shape stimuli (which are irrelevant) must also be automatically processed, leading to interference in performing the word to picture match. Thus interference results in tasks where the form of encoding of the relevant stimulus attribute must be transformed in order to make the response, in the presence of a competing attribute that is already close to the required form (Treisman \& Fearnley, 1969). This process is schematically illustrated in Figure 2. In across-attribute tasks, the to-be-ignored attribute automatically activates its memory representation, and because it is already close to the desired form of encoding, competes with the relevant attribute in generating the appropriate response. For within-attribute tasks, little or no inter-
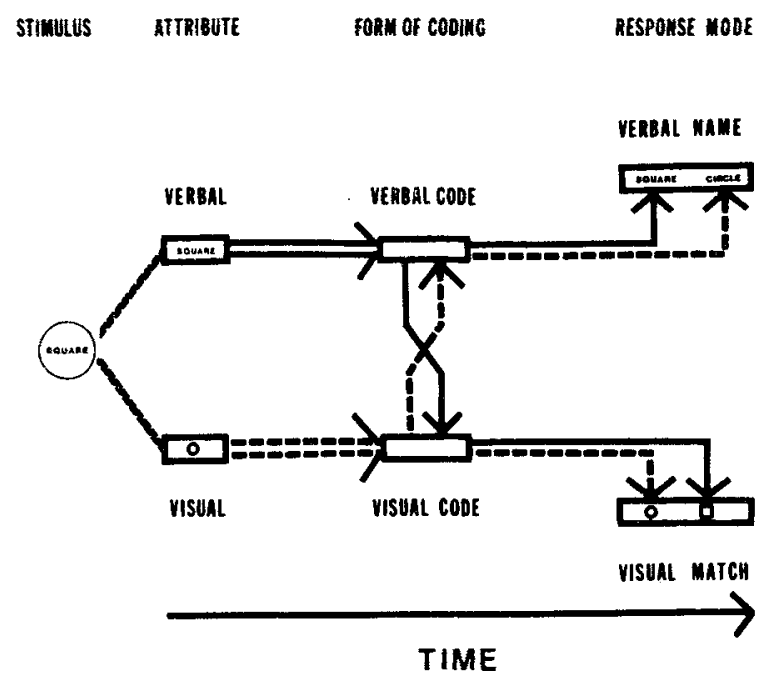

Figure 2. A schematic-diagram representing processing in tasks employing visual-verbal stimuli. The occurrence of interference will depend on both what stimulus attribute is relevant and what the response requirements are.

ference typically occurs, since the potentially interfering stimulus information is not in the appropriate form of coding to produce response competition. For example, if the verbal attribute "square" was to be matched to a picture of a square (as in Experiment 2), interference would occur, whereas if the circle was to be matched to a picture no interference would presumably occur.

According to traditional information processing views, there are two alternative ways of explaining exactly how the interference occurs. First, the automatic initiation of processing of the irrelevant attribute may somehow serve to block or delay the transformation of the relevant attribute into the appropriate form of coding prior to the stage of processing at which the response is generated. This assumes that the processing of the incongruent attribute (which is automatically activated) must at some point share limited processing resources with the relevant attribute information such that both cannot be processed as efficiently as would be the case alone.

The second alternative is that the interference may result from a form of response competition between the incongruent attribute and transformed attribute at the time the response is being generated. According to this view, the time course for these events favors the incongruent attribute as it is already close to the desired form of coding. The process of transforming the relevant stimulus attribute must necessarily slow down the total processing time for that attribute (i.e., "more" processing is required), such that both the incongruent and relevant attributes would be available in a pictorial form of representa- 
tion at the point where the motor code for responding is initiated. The presence of both attributes in a pictorial form of representation and the earlier availability of the incongruent attribute would thus produce a tendency to make the wrong responses which would have to be overcome. Either of these alternatives would presumably result in longer reaction times for stimuli with an incongruent pictorial attribute present. The present experiments offer no basis for distinguishing between these two alternatives.

At a broader level, both the encoding delay and response competition interpretations can be characterized as resource-limited processes (Norman \& Bobrow, 1975); the major distinction between them is simply the "level" at which they occur. What is more interesting, however, is that the interference is a transitory effect. The interference effects, which were very pronounced at the short delays between the word and pictorial matching field, were substantially reduced with a delay of $300 \mathrm{msec}$. This effect of the delay interval on the reaction times suggest that the incongruent attribute produces a transitory pathway activation that decays rapidly over time. Thus, with a sufficiently long delay, little or no interference is produced by the activation of the competing attribute, even though it is already in the form of encoding necessary for making the response. $^{5}$

Despite the obvious differences between the dimensions used in these experiments and that of color, and the fact that these differences seem to produce differing amounts of interference in the traditional Stroop task, the attenuation of interference with the longer delay intervals is the same as that found for color (Flowers, 1975). Thus, Dyer's claim (1973, p. 109) that the processing of color information differs from the processing of information about other dimensions does not appear to generalize to the present task. It is possible that the naming of colors is somehow slower than the naming of the attributes of other dimensions such as numerosity, direction, and achromatic shade, leading to the observed differences in the amount of interference produced by the presence of an incongruent attribute name. Morton and Chambers (1973) have come to a similar position in suggesting that the amount of interference that will result depends on the kind of attribute being named. For the present task, it appears that the process of generating a pictorial encoding of a verbal stimulus is similar for the dimensions of color, achromatic shade, and geometric shape.

One problem not yet considered is why the results for the congruent condition in Experiment 2 failed to show a facilitation effect. For example, when the word "SQUARE" was presented surrounded by a picture of a square, the RTs should have been faster than when the word was presented alone. That this did not occur may be seen as evidence supporting the perceptual delay model for the interference effect. If the interference from an incongruent attribute results from a delay in the transformation of the relevant attribute's form of encoding as a consequence of limited processing resources at this stage, then it is reasonable to expect that any time there are multiple sources of stimulus information which share limited processing resources, the processing of the relevant information will be slowed.

The lack of facilitation from the congruent shapes is in contrast to the suggestion by Posner and Snyder (1975a) that facilitation will occur in situations where the irrelevant information is congruent. Posner and Snyder base their conclusions on the results of a number of studies directed at assessing the effects of a prime (congruent or irrelevant) on matching latencies (Posner \& Snyder, 1975b). A major difference between their experiments and those reported here is that in the priming experiment the irrelevant information is presented prior to the relevant information, in a sequential fashion, whereas the present experiments presented both irrelevant and relevant information simultaneously. That the timing is critical can be seen in a study by Rosch (1975), where, in a matching task, she varied the interval between prime and stimuli and found that simultaneous presentation eliminated the facilitating effects of the prime.

One final implication of the present results concerns the Posner and Snyder (1975a) theory of automatic processing. Their discussion deals almost exclusively with evidence demonstrating that irrelevant "verbal" items, when closely related to the required tasks, are automatically processed by the subjects. Clearly, the data reported here illustrate that when an irrelevant pictorial attribute is pertinent to the task, automatic processing will occur, just as it does with words or letters.

\section{REFERENCES}

Clark, H. H., \& Brownell, H. H. Judging up and down. Journal of Experimental Psychology: Human Perception and Performance, 1975, 1, 339-352.

DYER, F. N. A comparison of chromatic and achromatic versions of the Stroop color-word test. Psychonomic Science, 1971, 22, 235-237.

DYER, F. N. The duration of word meaning responses: Stroop interference for different preexposures of the word. Psychonomic Science, 1971, 25, 229-231.

DYER, F. N. The Stroop phenomenon and its use in the study of perceptual, cognitive, and response processes. Memory \& Cognition, 1973, 1, 106-120.

Dyer, F. N., \& Severance, L. J. Effects of irrelevant colors on 
reading of color names: A controlled replication of the "reversed Stroop" effect. Psychonomic Science, 1972, 28, 336-338.

Egeth, H. E., Blecker, D. L., \& Kamlet, A. S. Verbal interference in a perceptual comparison task. Perception \& Psychophysics, 1969, 6, 355-356.

FLowERS, J. H. "Sensory" interference in a word-color matching task. Perception \& Psychophysics, 1975, 18, 37-43.

Flowers, J. H., \& Stoup, C. M. Selective attention between words, shapes, and colors in speeded classification and vocalization tasks. Memory \& Cognition, 1977, 5, 340-346.

Fraisse, $\mathbf{P}$. Why is naming longer than reading? Acta Psychologica, 1969, 30, 96-103.

GuMENIK, W. E., \& Glass, R. Effects of reducing the readability of the words in the Stroop color-word test. Psychonomic Science, $1970,20,247-248$.

Jensen, A. R., \& Rohwer, W. D., JR. The Stroop color-word test: A review. Acta Psychologica, 1966, 25, 36-93.

Morton, J. Categories of interference: Verbal mediation and conflict in card sorting. British Journal of Psychology, 1969, 60, 329-346.

Morton, J., \& Chambers, S. Selective attention to words and colours. Quarterly Journal of Experimental Psychology, 1973, 25, 387.397.

Norman, D. A., \& Bobrow, D. G. On data-limited and resourcelimited processes. Cognitive Psychology, 1975, 7, 44-64.

Posner, M. I., Boies, S. J., Eichelman, W. H., \& Taylor, R. L. Retention of visual and name codes of single letters. Joumal of Experimental Psychology Monograph, 1969, 79, 1-13.

PosNer, M. I., \& SNYDER, C. R. R. Attention and cognitive control. In R. L. Solso (Ed.), Information processing and cognition. Hillsdale, N.J: Erlbaum, 197S. (a)

PosNer, M. I., \& SNYDER, C. R. R. Facilitation and inhibition in the processing of signals. In P. M. A. Rabbit (Ed.), Attention and performance V. London: Academic Press, 1975. (b)

Roscr, E. The nature of mental codes for color categories. Journal of Experimental Psychology: Human Perception and Performance, 1975, 1, 303-322.

SHOR, R. E. The processing of conceptual information on spatial directions from pictorial and linguistic symbols. Acta Psychologica, 1970, 32, 346-365.

STROOP, J. R. Studies of interference in serial verbal teactions. Journal of Experimental Psychology, 1935, 18, 643-662.

Treisman, A. M., \& Fearnley, S. The Stroop test: Selective attention to colours and words. Nature, 1969, 222, 437-439.

TVERSKx, B. Pictorial and verbal encoding in a short term memory task. Perception \& Psychophysics, 1969, 6, 225-233.

Uleman, J. S., \& Reeves, J. A. A reversal of the Stroop interference effect, through scanning. Perception \& Psychophysics, $1971,9,293-295$.

WARREN, R. E., \& LASHER, M. D. Interference in a typeface variant of the Stroop test. Perception \& Psychophysics, 1974, $15,128-130$

White, B. W. Interference in identifying attributes and attribute names. Perception \& Psychophysics, 1969, 6, 166-168.

\section{NOTES}

1. We use the phrase "pictorial attribute" to refer to the visual properties of a stimulus, such as the ink color in which word is written, the shape of the letters that compose a word, or the location of a target word on a field, etc.

2 . In one condition where the legibility of the words was severely reduced by the use of a mask, Dyer found just a $20 \%$ increase in response time, still nowhere near comparable to the normal Stroop effect.

3. A control condition, the equivalent of presenting the word alone without background shade, is clearly impossible for this task. The letters of the word and the background on which they are printed both must have some value on the dimension of achromatic shade.

4. Though the differences were small $(15,1$, and $10 \mathrm{msec}$ for the $20-, 100-$, and 300 -msec delays, respectively), at all three delays the word alone condition produced faster average response times than did the congruent condition. It is possible that this difference would have approached significance with extended trials. If so, it would appear that the presence of any irrelevant material increases RT-congruent information only slightly, incongruent information to a greater degree.

5 . This reduction in interference with longer delays would be expected whether the interference was assumed to be due to a delay in the transformation of the form of encoding of the verbal attribute or due to response competition. In the former case, when a sufficiently long delay interval is provided, presumably the encoding transformation (though delayed) would be completed in time so as to not produce interference, while in the latter case, if the automatic processing of the irrelevant attribute is only transitory, then after a long delay the processing trace would be expected to have decayed sufficiently so that it would not interfere with the intentional processing of the relevant attribute into the appropriate motor code for response output. Support for this interpretation is provided by Dyer (1971) in a study on the Stroop effect in which he preexposed the color word in black ink prior to presenting the color. The longer the delay between the preexposed word and the color, the less interference was produced in the naming response.

It is conceivable that the decrease in interference that occurred at the 300 -msec delay was due to a ceiling effect that prevented any further increase in RT for the word alone or congruent conditions. However, the RT data contradict this interpretation. Examination of the RTs for the congruent stimulus conditions in both experiments at the $300-\mathrm{msec}$ delay shows a difference of $40 \mathrm{msec}$. Thus, for Experiment 2 there was no ceiling effect. Since the pattern of results for the two experiments is essentially the same, it seems doubtful that a ceiling effect was a factor in the first experiment either.

(Received for publication February 21, 1977; revision accepted June 14, 1977.) 